\title{
Some Properties of Topological Spaces Related to the Local Density and the Local Weak Density
}

\author{
Beshimov R.B. ${ }^{1, *}$, Mamadaliev N.K. ${ }^{2}$, Mukhamadiev F.G. ${ }^{1}$ \\ ${ }^{1}$ Department of Mathematics, Tashkent State Pedagogical University named after Nizami, \\ str. Yusuf Khos Hojib 103, 100070 Tashkent, Uzbekistan \\ ${ }^{2}$ Institute of Mathematics, National University of Uzbekistan named after Mirzo Ulugbek, \\ Durmon yuli str. 29, 100125 Tashkent, Uzbekistan
}

Copyright (C) 2015 by authors, all rights reserved. Authors agree that this article remains permanently open access under the terms of the Creative Commons Attribution License 4.0 International License

\begin{abstract}
In the paper the local density and the local weak density of topological spaces are investigated. It is proved that for stratifiable spaces the local density and the local weak density coincide, these cardinal numbers are preserved under open mappings, are inverse invariant of a class of closed irreducible mappings. Moreover, it is showed that the functor of probability measures of finite supports preserves the local density of compacts.
\end{abstract}

Keywords Local Density, Local Weak Density

AMS Subject Classification: 54A25, 54B20

\section{Introduction}

The local properties play an important role in general topology. For instance, compactness in $R^{n}$ is equivalent to total boundedness and closedness. In the case of local compactness boundedness is not necessary. In a case of locality some properties can be lost or some new properties may appear. For example, an open subspace of a compact space may often be non-compact. But any open subspace of a locally compact space is locally compact.

In the paper we consider the local density and the local weak density of topological spaces. We investigate what properties are preserved in a local cases or what properties may appear.

It is known that the density (the weak density) is preserved under continuous onto mappings, is hereditary with respect to $F_{\sigma}$-sets and closed domains. The continuum product of separable (weakly separable) spaces is separable (weakly separable, respectively), any compact extension of a weakly separable space is weakly separable.

It turns out that the local density (the local weak density) is not preserved under a continuous mapping (example 2.1), a compact extension of a locally separable (locally weakly separable) space can be not locally separable (locally weakly separable, respectively) (example 2.2), the product of infinitely many locally separable (locally weakly separable) spaces is not always locally separable (respectively, locally weakly separable) (example 2.3).

Recall some definitions and propositions related to the work.

Definition 1.1[1]. A subset $M$ of a topological space $X$ is dense in $X$ if $[M]=X$.

The density of a topological space is defined with following way:

$$
d(X)=\min \{|M|: M \text { is dense in } X\} .
$$

If $d(X) \leq \aleph_{0}$ for a space $X$ we say that $X$ is separable.

Definition 1.2[2]. The weak density of a topological space $X$ is the smallest cardinal number $\tau \geq \aleph_{0}$ such that there is a $\pi$-base in $X$ coinciding with $\tau$ centered systems of open sets, i.e. there is a $\pi$-base $\mathcal{B}=\bigcup\left\{B_{\alpha}\right.$ : $\alpha \in X\}$, where $B_{\alpha}$ is a centered system of open sets for each $\alpha \in A$ and $|A|=\tau$.

The weak density of a topological space $X$ is denoted by $w d(X)$. If $w d(X) \leq \aleph_{0}$ then we say that a topological space $X$ is weakly separable.

Definition 1.3[3]. A topological $T_{1}$-space $X$ is a stratifiable space if to each open set $U \subseteq X$, one can assign a sequence of open sets $\left\{U_{n}: n \in N\right\}$ of the space $X$ such that
a) $\left[U_{n}\right] \subset U$;
b) $\bigcup\left\{U_{n}: n \in N\right\}=U$;
c) $U_{n} \subseteq V_{n}$, whenever $U \subseteq V$.

It is clear that every metrizable space is stratifiable.

Definition 1.4 [1]. A continuous mapping $f: X \rightarrow$ $Y$ of a topological space $X$ into a topological space $Y$ is called open (closed) if for each open (closed) set $A \subset X$ the image $f(A)$ is open (closed) in $Y$.

Theorem 1.1[3]. The stratifiability is hereditary property, i.e. every subspace of a stratifiable space is stratifiable. 
Theorem 1.2[2]. For any stratifiable space $X$ we have $w d(X)=d(X)$.

Theorem 1.3[2]. If $w d(X) \leq \tau$ and $f: X \rightarrow Y$ is a continuous mapping of $X$ onto $Y$, then $w d(Y) \leq \tau$.

Theorem 1.4[1]. If $d(X) \leq \tau$ and $f: X \rightarrow Y$ is a continuous mapping of $X$ onto $Y$, then $d(Y) \leq \tau$.

Proposition 1.1[1]. If $d(X) \leq \tau$ and $G$ is an arbitrary non-empty open subset of the space $X$, then $d(G) \leq \tau$.

Proposition 1.2[4]. If $w d(X) \leq \tau$ and $G$ is an arbitrary non-empty open subset of the space $X$, then $w d(G) \leq \tau$.

\section{Main results}

Definition 2.1. We say that a topological space $X$ is locally $\tau$-dense at a point $x \in X$ if $\tau$ is the smallest cardinal number such that $x$ has a $\tau$-dense neighborhood in $X$.

The local density at a point $x$ is denoted by $l d(x)$.

The local density of a space $X$ is defined as the supremum of all numbers $l d(x)$ for $x \in X$; this cardinal number is denoted by $l d(X)$.

If $l d(X) \leq \aleph_{0}$ for a space $X$, we say that $X$ is locally separable.

Definition 2.2. A topological space $X$ is locally weakly $\tau$ dense at a point $x \in X$ if $\tau$ is the smallest cardinal number such that $x$ has a neighborhood of weak density $\tau$ in $X$.

The local weak density at a point $x$ is denoted by $l w d(x)$.

The local weak density of a topological space $X$ is defined with following way:

$$
\operatorname{lwd}(X)=\sup \{\operatorname{lwd}(x): x \in X\} .
$$

If $l w d(X) \leq \aleph_{0}$ for a space $X$, then we say that $X$ is locally weakly separable [4].

From the work [4] it is easy to obtain the followings

Proposition 2.1. Let lwd $(X) \leq \tau$ and let $f: X \rightarrow Y$ be an open mapping of $X$ onto $Y$. Then lwd $(Y) \leq \tau$.

Theorem 2.1. Suppose a topological $T_{1}$-space $X$ is one of the followings:

1) Pseudometric;

2) Locally compact;

3) Linear ordered,

then $l d(X)=l w d(X)$.

Theorem 2.2. Let $X$ be a locally weakly $\tau$-dense space and let $G$ be its subspace satisfying one of the following conditions:

1) $G$ is open in $X$;

2) $G$ is dense in $X$;

3) $G$ is a closed domain in $X$,

then $G$ is locally weakly $\tau$-dense.

Theorem 2.3. For any stratifiable space $X$ we have $l d(X)=l w d(X)$.

Proof. 1) It is clear that the inequality $\operatorname{lwd}(X) \leq$ $l d(X)$ holds for any topological $T_{1}$-space $X$.

2) We shall show $l d(X) \leq l w d(X)$. Let $l w d(X)=\tau$. Then for any point $x \in X$ there exists a neighborhood $O x$ of $x$ such that $w d(O x)=\tau$. Since $X$ is stratifiable, by theorem 1.2 the subspace $O x$ is also stratifiable. Then by theorem 1.2 we have $d(O x)=w d(O x)=\tau$. For an arbitrary point $x \in X$ we have found the neighborhood $O x$ of density $\tau$. This means that $l d(X) \leq \tau=l w d(X)$. Theorem 2.3 is proved.

Remark 2.1. The following example shows that in proposition 1.1 openness of a mapping $f$ is important. There is a continuous mapping $f$ which does not preserve the local weak density of topological spaces.

Example 2.1. Let $\left(R, \tau_{1}\right)$ be the real line with the discrete topology and let $\left(R, \tau_{2}\right)$ be the real line with the natural topology. Consider identity mapping from $\left(R, \tau_{1}\right)$ onto $\left(R, \tau_{2}\right)$, i.e. $i d:\left(R, \tau_{1}\right) \rightarrow\left(R, \tau_{2}\right)$.

Since $\left(R, \tau_{1}\right)$ is discrete space, every mapping, in particular, id is continuous. Moreover, each point $x \in R$ has one-point neighborhood $\{x\}$. This implies

$$
1=\operatorname{ld}\left(R, \tau_{1}\right)=\operatorname{lwd}\left(R, \tau_{1}\right) \underset{\aleph_{0} .}{<} \operatorname{ld}\left(R, \tau_{2}\right)=\operatorname{lwd}\left(R, \tau_{2}\right)=
$$

Definition 2.3[1]. A closed mapping $f: X \rightarrow Y$ is perfect, if $f^{-1}(y)$ is compact for every point $y \in Y$.

Theorem 2.4. Let $f$ be a perfect mapping of the space $X$ onto the space $Y$. Then

1) $l d(Y) \leq l d(X)$

2) $l w d(Y) \leq l w d(X)$.

Proof. 1) We shall prove $l w d(Y) \leq l w d(X)$. Let $\operatorname{lwd}(X)=\tau \geq \aleph_{0}$. Let us take an arbitrary point $x \in X$. Then the set $f^{-1}(y) \subset X$ is compact in $X$. For every point $x \in f^{-1}(y)$ there exists a neighborhood $O x$ of $x$ such that $w d(O x) \leq \tau$. The family of all these neighborhoods covers the set $f^{-1}(y)$. Since $f^{-1}(y)$ is compact, there is a finite sequence $O x_{1}, O x_{2}, \ldots, O x_{n}$ of open sets such that $f^{-1}(y) \subset \bigcup_{i=1}^{n} O x_{i}$ and $w d\left(O x_{i}\right) \leq \tau$ for each $i=1,2, \ldots, n$. Put $G=\bigcup_{i=1}^{n} O x_{i}$. Then we obtain $w d(G) \leq \tau$ and $X \backslash G \subset X \backslash f^{-1}(y)$. This implies $f(X \backslash G) \subset f\left(X \backslash f^{-1}(y)\right)$.

Let us show now $y \notin f\left(X \backslash f^{-1}(y)\right)$. Suppose it does not hold, i.e. $y \in f\left(X \backslash f^{-1}(y)\right)$. In this case, there is a point $x \in X \backslash f^{-1}(y)$ such that $f(x)=y$. But it is impossible, since $x \notin f^{-1}(y)$. Therefore $y \notin$ $f\left(X \backslash f^{-1}(y)\right)$ and, a fortiori, $y \notin f(X \backslash G)$. The set $f(X \backslash G)$ is closed as the image of a closed set under a closed mapping. Hence, the set $Y \backslash f(X \backslash G)$ is an open set containing $y$.

We shall show $Y \backslash f(X \backslash G) \subset f(G)$. Indeed, since $X \backslash G \subset f^{-1}(f(X \backslash G))$, we obtain $f^{-1}(Y \backslash f(X \backslash G))=$ $X \backslash f^{-1}(f(X \backslash G)) \subset X \backslash(X \backslash G)=G$. This implies $Y \backslash f(X \backslash G) \subset f(G)$. Therefore $Y \backslash f(X \backslash G)$ is an open subset of $f(G)$. Since $w d(G) \leq \tau$, by theorem 1.3 we obtain $w d(f(G)) \leq \tau$. Then by proposition 1.2 we have $w d(Y \backslash f(X \backslash G)) \leq \tau$. Thereby, we have found the neighborhood $Y \backslash f(X \backslash G)$ of weak density $\leq \tau$ for arbitrarily chosen point $y \in Y$. Therefore $l w d(Y) \leq \tau$. The inequality $l w d(Y) \leq l w d(X)$ is proved.

2) Let us show now $l d(Y) \leq l d(X)$. Let $l d(X)=$ $\tau \geq \aleph_{0}$. Take an arbitrary point $y \in Y$. Then the set $f^{-1}(y) \subset X$ is compact in $X$. For each point $x \in f^{-1}(y)$ there exists a neighborhood $O x$ of $x$ such that $d(O x) \leq \tau$. The family of all these neighborhoods covers the set $f^{-1}(y)$. Since $f^{-1}(y)$ is compact, there is a finite sequence $O x_{1}, O x_{2}, \ldots, O x_{n}$ open sets such that $f^{-1}(y) \subset \bigcup_{i=1}^{n} O x_{i}$ and $d\left(O x_{i}\right) \leq \tau$ for each $i=1,2, \ldots, n$. 
Put $G=\bigcup_{i=1}^{n} O x_{i}$. Then we obtain $d(G) \leq \tau$ and $X \backslash G \subset$ $X \backslash f^{-1}(y)$. This implies $f(X \backslash G) \subset f\left(X \backslash f^{-1}(y)\right)$.

As it was shown in 1$), Y \backslash f(X \backslash G)$ is an open set containing $y$ and the inclusion $Y \backslash f(X \backslash G) \subset f(G)$ holds. Since $d(G) \leq \tau$, by theorem 1.4 we see that $d(f(G)) \leq \tau$. Then by proposition 1.1 we have $d(Y \backslash f(X \backslash G)) \leq \tau$ and we have found the neighborhood of density $\leq \tau$ for an arbitrary chosen point $y \in Y$. Therefore $l d(Y) \leq \tau$. The inequality $l d(Y) \leq l d(X)$ is proved. Theorem 2.4 is proved.

Definition 2.4[5]. A continuous mapping $f: X \rightarrow Y$ of a space $X$ onto a space $Y$ is irreducible if $f(A) \neq Y$ for any proper closed subset $A$ of the space $X$.

Theorem 2.5. Let $f$ be a closed and irreducible mapping of a space $X$ onto a space $Y$. Then

1) $l w d(X) \leq l w d(Y)$

2) $l d(X) \leq l d(Y)$.

Proof. 1) Let us show firstly $l w d(X) \leq l w d(Y)$. Let $\operatorname{lwd}(Y)=\tau \geq \aleph_{0}$. Take an arbitrary point $x \in X$, then $f(x) \in Y$. Since $l w d(Y)=\tau$, there exists a neighborhood $O f(x)$ of the point $f(x)$ in $Y$ such that $w d(O f(x)) \leq \tau$. This means that there is a $\pi$-base $\beta=\cup\left\{\beta_{\alpha}: \alpha \in A\right\}$ in $O f(x)$, where $|A| \leq \tau$ and $\beta_{\alpha}=\left\{U_{s}^{\alpha}: s \in A_{\alpha}\right\}$ is a centered system of open sets in $O f(x)$ for every $\alpha \in A$. Then it is clear that $f^{-1}\left(\beta_{\alpha}\right)=$ $\left\{f^{-1}\left(U_{s}^{\alpha}\right): s \in A_{\alpha}\right\}$ is a centered system of open sets in the neighborhood $f^{-1}(O f(x))$ of $x$. This implies that the system $f^{-1}(\beta)=\cup\left\{f^{-1}\left(\beta_{\alpha}\right): \alpha \in A\right\}$ coincides with $\tau$ centered systems of open sets in $f^{-1}(O f(x))$. Now we shall show that $f^{-1}(\beta)$ is a $\pi$-base in $f^{-1}(O f(x))$.

Let $G$ be a nonempty open subset of $f^{-1}(O f(x))$. Since $f$ is closed and irreducible, the set $f(X \backslash G)$ is closed in $Y$ and $f(X \backslash G) \neq Y$. Then $Y \backslash f(X \backslash G)$ is a nonempty open subset of the space $Y$.

Now we shall show $Y \backslash f(X \backslash G) \subset f(G)$. Indeed $f^{-1}(Y \backslash f(X \backslash G))=X \backslash f^{-1}(f(X \backslash G)) \subset X \backslash(X \backslash G)=G$. This implies $Y \backslash f(X \backslash G) \subset f(G)$.

On the other hand $f(G) \subset O f(x)$. Hence, $Y \backslash f(X \backslash G)$ is a nonempty open subset of $O f(x)$. Since $\beta$ is a $\pi$ base in $O f(x)$, there exists an element $U_{s}^{\alpha} \in \beta$ such that $U_{s}^{\alpha} \subset Y \backslash f(X \backslash G)$ Thence, we have $f^{-1}\left(U_{s}^{\alpha}\right) \subset$ $f^{-1}(Y \backslash f(X \backslash G))=X \backslash f^{-1}(f(X \backslash G)) \subset X \backslash(X \backslash G)=G$. Therefore $f^{-1}(\beta)$ is a $\pi$-base in $f^{-1}(O f(x))$. Hence $w d\left(f^{-1}(O f(x))\right) \leq \tau$. Since the point $x$ was chosen arbitrarily, we obtain $l w d(X) \leq \tau$.

2) Let us prove now $l d(X) \leq l d(Y)$. Let $l d(Y)=\tau \geq$ $\aleph_{0}$. We have to show $l d(X) \leq \tau$. Take an arbitrary point $x \in X$, then $f(x) \in Y$. Since $l d(Y)=\tau$, there exists a neighborhood $O f(x)$ of $f(x)$ in $Y$ such that $d(O f(x)) \leq$ $\tau$. This means that there is a dense subset $M=\left\{y_{\alpha}\right.$ : $\alpha \in A\}$ of the subspace $O f(x)$ such that $|A| \leq \tau$. Let us choose a point $x_{\alpha}$ from each set $f^{-1}\left(y_{\alpha}\right)$ and form the set $M_{1}=\left\{x_{\alpha}: \alpha \in A\right\}$. It is clear that $\left|M_{1}\right|=\tau$. Let us show that $M_{1}$ is dense in $f^{-1}(O f(x))$.

Let $G$ be an arbitrary nonempty subset of $f^{-1}(O f(x))$. Then $f(G) \subset f(O f(x))$. Since $f$ is closed and irreducible, the set $f(X \backslash G)$ is closed in $Y$ and $f(X \backslash G) \neq Y$. As it was shown in 1$), Y \backslash f(X \backslash G)$ is a nonempty open set of the space $Y$ and $Y \backslash f(X \backslash G) \subset$ $f(G)$. Therefore, $Y \backslash f(X \backslash G)$ is nonempty open subset of $O f(x)$. Since $M$ is dense in $O f(x)$, there exists an element $y_{\alpha} \in M \cap Y \backslash f(X \backslash G)$. This implies $f^{-1}\left(y_{\alpha}\right) \subset$
$f^{-1}(Y \backslash f(X \backslash G))=X \backslash f^{-1}(f(X \backslash G)) \subset X \backslash(X \backslash G)=$ $G$. Then by choice of the elements of $M_{1}$ we have $x_{\alpha} \in f^{-1}\left(y_{\alpha}\right) \subset G$. This means that $M_{1}$ is dense in $f^{-1}(O f(x))$. Therefore $d\left(f^{-1}(O f(x))\right) \leq \tau$ and - the point $x$ being arbitrary $-l d(X) \leq \tau$. Theorem 2.5 is proved.

From theorems 2.4 and 2.5 we directly obtain the following

Theorem 2.6. Let $f$ be a perfect and irreducible mapping of a space $X$ onto a space $Y$. Then $\operatorname{lwd}(Y)=$ $l w d(X)$ and $l d(X)=l d(Y)$.

In the work [6] shown that following example:

Example 2.2[6]. Let $M=R^{2}(x, y)$ be the Euclidean plane with the Cartesian coordinates $(x, y)$. Leaves $L_{\alpha}$ of foliation $F$ are given by the equations $y=\alpha=$ const. Then the plan homeomorphism $\varphi: R^{2} \rightarrow R^{2}$ determined by the formula $\varphi(x, y)=\left(x+y, y^{\frac{1}{3}}\right)$ is an isometry of the foliation $F$, but is not a diffeomorphism of the plane.

Every leave of the foliation, in example 2.2 is locally separable.

Let $X$ be a compact space. By $C(X)$ denote the set of all continuous maps $\phi: X \rightarrow R$ with the usual sup-norm $\|\phi\|=\sup \{|\phi(x)|: x \in X\}$. A continuous functional $\mu: C(X) \rightarrow R$ is called a measure on the compact $X$. A measure is positive (notation $\mu \geq 0$ ), if $\mu(\phi) \geq 0$ for any $\phi \geq 0$. A measure is normed, if $\|\mu\|=1$. A positive normed measure is called a probability measure. A space consisting of all probability measures, denote by $P(X)$. A neighborhood base at a point $\mu \in P(X)$ consists of all the sets in the form

$$
\begin{gathered}
O\left(\mu ; \phi_{1}, \phi_{2}, \ldots, \phi_{k} ; \varepsilon\right)=\left\{\nu \in P(X):\left|\mu\left(\phi_{i}\right)-\nu\left(\phi_{i}\right)\right|<\right. \\
\varepsilon, i=1,2, \ldots, k\}
\end{gathered}
$$

where $\phi_{1}, \phi_{2}, \ldots, \phi_{k} \in C(X)$ and $\varepsilon>0$.

A support $\operatorname{supp}(\mu)$ of a measure $\mu \in P(X)$ is the smallest closed subset $F \subset X$ such that $\mu(F)=\mu(X)$. For a compact $X$ and a natural number $n$ put $P_{n}(X)=$ $\{\mu \in P(X):|\operatorname{supp}(\mu)| \leq n\}[7]$.

Proposition 2.2[4]. Let $X$ be a topological $T_{1}$-space. If $l d(X)=\tau \geq \aleph_{0}$ then $l d\left(X^{n}\right)=\tau$ for any $n \in N$.

Theorem 2.7. Let $X$ be an infinite compact space. Then $l d\left(P_{n}(X)\right) \leq l d(X)$.

Proof. Let $X$ be an infinite compact space and let $l d(X)=\tau \geq \aleph_{0}$. Then $l d\left(X^{n}\right)=\tau$ by proposition 2.2 . In [8] V.Basmanov showed that the space $P_{n}(X)$ can be represented as a continuous image of the space $X \times$ $\sigma^{n-1}$, where $\sigma^{n-1}$ is the $(n-1)$-dimensional simplex. The mapping $\pi: X \times \sigma^{n-1} \rightarrow P_{n}(X)$ is defined by the formula

$$
\pi\left(x_{1}, \ldots, x_{n}, m_{1}, \ldots, m_{n}\right)=\sum_{i=1}^{n} m_{i} \delta_{x_{i}}
$$

where $\left(m_{1}, \ldots m_{n}\right) \in \sigma^{n-1}, \sum_{i=1}^{n} m_{i}=1$ and $m_{i} \geq 0$ for each $i \in N, \delta_{x_{i}}$ are Dirak's measures at points $x_{i}$ respectively. The mapping $\pi$ is perfect, since $\pi$ is continuous mapping defined on compact $X \times \sigma^{n-1}$. Then by theorem 2.4 we have $l d\left(P_{n}(X)\right) \leq \tau$. Theorem 2.7 is proved.

Let us recall now P.S.Alexandroff's one-point compactification. Let $X$ be an uncountable set and $\xi$ is a point which does not belong to $X$. Define a topology on 
the set $X \cup\{\xi\}$ with a base. The family of all the onepoint subsets of $X$ and all the sets of the form $X \backslash A$, where $A$ is a finite subset of $X$, forms a base of topology on $X \cup\{\xi\}$. This space is denoted by $\alpha X$. Clearly, the space $\alpha X$ is compact and Hausdorff.

Remark 2.1. There exist a topological space $Y$ and its dense subspace $X$ such that $l d(X)<l d(Y)$ and $l w d(X)<l w d(Y)$.

Example 2.3. Let $X$ be the discrete space of cardinality $\tau=c$ (continuum). It is clear that $l d(X)=$ $1<\aleph_{0}$ ( $\aleph_{0}$ is the infinite countable cardinal). Consider P.S.Alexandroff's one-point compactification of $X$. This compactification denote by $Y$. Then $Y$ is compact and $l d(Y) \geq \tau$.

Indeed $Y=X \cup\{\xi\}$, where $\xi \notin X$. Then for every neighborhood of the point $\xi$ we have $d(O \xi)=c$, since $|Y \backslash O \xi|<\aleph_{0}$ is finite. Therefore $1=l d(X)<l d(Y)=c$. Analogously, we can prove that $1=\operatorname{lwd}(X)<\operatorname{lwd}(Y)=$ c.

Theorem 2.8[2]. Let $X$ be a topological $T_{1}$-space. If $w d\left(X_{s}\right) \leq \tau$ for every $s \in S$ and $|S| \leq 2^{\tau}$, then $w d\left(\prod_{s \in S} X_{s}\right) \leq \tau$.

Theorem 2.9. Let $\tau$ be an infinite cardinal number. Consider a family of topological spaces $\left\{X_{s}: s \in S\right\}$, where $|S| \leq 2^{\tau}$. If every space $X_{s}$ is locally weakly $\tau$ dense and there exists a finite subset $S_{0}$ of the index set $S$ such that $X_{s}$ is weakly $\tau$-dense for all $s \in S \backslash S_{0}$, then the product $\prod_{s \in S} X_{s}$ is locally weakly $\tau$-dense.

Proof. Take an arbitrary point $x=\left\{x_{s}: s \in S\right\}$ from the product $\prod_{s \in S} X_{s}$. Since all the spaces $X_{s}$ are locally weakly $\tau$-dense, the point $x_{s} \in X_{s}$ has a neighborhood $U_{s}$ of weak density $\leq \tau$ for every $s \in S_{0}$. The set $\prod_{s \in S_{0}} U_{s} \times \prod_{s \in S \backslash S_{0}} X_{s}$ is a neighborhood of the point $x$ in $\prod_{s \in S} X_{s}$. Since $d\left(X_{s}\right) \leq \tau$ for every $s \in S \backslash S_{0}$, by theorem 2.8 we have $d\left(\prod_{s \in S_{0}} U_{s} \times \prod_{s \in S \backslash S_{0}} X_{s}\right) \leq \tau$. So, we have found a weakly $\tau$-dense neighborhood of the point $x$ in $\prod_{s \in S} X_{s}$. The point $x$ was chosen arbitrarily, therefore the product $\prod_{s \in S} X_{s}$ is locally weakly $\tau$-dense. Theorem 2.9 is proved.

Corollary 2.1. Consider the family of topological spaces $\left\{X_{s}: s \in S\right\}$, where $|S| \leq 2^{\aleph_{0}}$. If all the spaces $X_{s}$ are locally weakly separable and there exists a finite subset $S_{0}$ of the index set $S$ such that $X_{s}$ is weakly separable for $s \in S \backslash S_{0}$, then the product $\prod_{s \in S} X_{s}$ is locally weakly separable.

It seems, the inverse statement is also true

Theorem 2.10. Suppose that the product $\prod_{s \in S} X_{s}$ is locally weakly $\tau$-dense. Then all the spaces $X_{s}$ are locally weakly separable, moreover, there exists a finite subset $S_{0}$ of the index set $S$ such that $X_{s}$ is weakly $\tau$-dense for every $s \in S \backslash S_{0}$.

Proof. Since all the projection $p_{s}: \prod_{s \in S} X_{s} \rightarrow X_{s}$ are open, from proposition 2.1 it directly follows that all the spaces $X_{s}$ are locally weakly $\tau$-dense. Let us now prove the second statement of the theorem. Take an arbitrary point $x=\left(x_{s}\right)_{s \in S}$ from the product $\prod_{s \in S} X_{s}$. Since the product $\prod_{s \in S} X_{s}$ is locally weakly $\tau$-dense, the point $x$ has a weakly $\tau$-dense neighborhood $\prod_{s \in S_{0}} U_{s} \times \prod_{s \in S \backslash S_{0}} X_{s}$ (since the weak density is hereditary with respect to open subsets, we can assume that the neighborhood is from the canonical base of $\prod_{s \in S} X_{s}$ ), where $S_{0}$ is a finite subset of $S$ such that $x_{s} \in U_{s}$ for $s \in S_{0}, x_{s} \in X_{s}$ for $s \in S \backslash S_{0}$. Since the weak density is preserved under open mappings and the projections are open maps, we see that $X_{s}$ is weakly $\tau$-dense for every $s \in S \backslash S_{0}$. Theorem 2.10 is proved.

Note that in the inverse statement the condition $|S| \leq$ $2^{\tau}$ is omitted.

Corollary 2.2. Suppose that the product $\prod_{s \in S} X_{s}$ is locally weakly separable. Then all the spaces $X_{s}$ are locally separable, moreover, there exists a finite subset $S_{0}$ of $S$ such that $X_{s}$ is separable for every $s \in S \backslash S_{0}$.

Remark 2.2. The following example shows that the local separability and the local weak separability are not preserved under the infinite product.

Example 2.4. Let $X$ be the discrete space of cardinality $c$. It is obvious that $l d(X)=\operatorname{lwd}(X)=$ $1<\aleph_{0}$, i.e. it is locally separable and locally weakly separable.

Now let us consider the product $X^{N}$ of countably many copies of the space $X$. Take an arbitrary point $x=\left(x_{i}\right)_{i \in N} \in X^{N}$ and consider it arbitrary canonical neighborhood $O x=\left\{x_{i_{1}}\right\} \times \ldots \times\left\{x_{i_{k}}\right\} \times X^{N^{\prime}}$, where $N^{\prime}=N \backslash\left\{i_{1}, i_{2}, \ldots, i_{k}\right\}$. It is clear that $O x$ is homeorphic to $X^{N}$. This implies that $d(O x)=d\left(X^{N}\right)=c$ and $w d(O x)=w d\left(X^{N}\right)=c$. We obtain the fact that the point $x$ has neither separable nor weakly separable neighborhood. Therefore $l d\left(X^{N}\right)>\aleph_{0}$ and $l w d\left(X^{N}\right)>$ $\aleph_{0}$, i.e. the product of countably many copies of the space $X$ is neither locally separable, nor locally weakly separable.

In the future works, we are planning to investigate cardinal invariants and functors preserving them. Now, we are trying to give answers to the following open questions: Does the functor $F$ preserve the tightness, the density, the weak density, the local density and the local weak density, where $F$ can be the exponential functor exp, the functor of superextension $\lambda$, the functor of probability measures $P$ and the functor of weakly additive functionals $O$ ?

\section{REFERENCES}

[1] R. Engelking, General topology, Moscow: Mir (1986), $752 \mathrm{p}$.

[2] R.B. Beshimov, On weakly density of topological spaces DAN RUz, 11 (2000), 10-13.

[3] J. Ceder, Some generalizations of metric spaces Pacific Journal of Mathematics, 11 (1961), 105-126.

[4] R.B. Beshimov, G.F. Djabbarov, On local weakly separable spaces Methods of Functional Analysis and Topology, 3 (2005), 217-221. 
[5] A.V. Arhangel'skii, V.I. Ponomarev, Fundamentals of general topology in problems and exersices, Moscow: Nauka (1974). 424 p.

[6] A.S. Sharipov, A.Ya. Narmanov, On the isometries of foliated manifold TWMS Jour. Pure. Appl. Math. V. 2, N. 1, (2011), pp. 127-133.
[7] V.V. Fedorchuk, V.V. Filippov, General topology. Basic constructions, Moscow: Fizmatlit (2006), 332 p.

[8] V.N. Basmanov, Covariant functors, retracts and dimension DAN SSSR, 271 (1983), 1033-1036.0 